\title{
Combined Action of Low-Intensity Physical Factors (Including Biologically Active Substances in Ultra-Low Doses) and Intensive Physical and Chemical Factors in Medicine
}

\author{
*Liudmila B Boldyreva \\ State University of Management, Russia
}

Submission: June 26, 2017; Published: June 28, 2017

"Correspondence Address: Liudmila B Boldyreva, State University of Management, 26 Bakinskih Comissarov Ulitsa 7-6-28, Moscow 119571, Russia, Email: boldyrev-m@yandex.ru

Abstract

A possibility of effective therapeutic action on biological objects of intensive physical and chemical factors jointly with biologically active substances in ultra-low doses and with low-intensity physical factors is discussed. The low-intensity physical factors are infra-low-frequency magnetic fields, the field-free magnetic vector potential, effect of cavity structures, quantum correlation of quantum entities.

Keywords: Ultra-low doses; Homeopathy; Magnetic vector potential; Cavity structures; Quantum correlation; Spin supercurrent

\section{Introduction}

To intensive physical and chemical factors used in medicine refer, in particular, intensive radiotherapy and chemotherapy, which are effective remedies for a lot of diseases and nearly all malignant tumors. However, these remedies have a disadvantage of negatively affecting healthy organs. One of the means to minimize the negative effect is the precise delivery of remedies to the diseased organ. Other method is the decreasing of doses of radiation and chemical agents due to combined action of these agents with low-intensity physical factors. It is shown in this work that such factors are infra-low-frequency magnetic fields, the field-free magnetic vector potential, energy of cavity structures, quantum correlation of quantum entities, biologically active substances in ultra-low doses (including doses used in homeopathy). The effect of all the above mentioned factors on biological objects takes place owing to their action on spins of virtual particles created by quantum entities that constitute the biological objects [1-7].

\section{Results and Discussion}

\section{Infra-low-frequency electromagnetic fields}

Biological systems are normally highly sensitive to lowfrequency, low-intensity electromagnetic fields. For example, the field of some $\mathrm{Hz}$ frequency has a considerable effect on the blood clotting time and modifies the sensitivity of nerve cells to hypoxia [1]. The studies conducted showed the advisability of using the low-intensity radiation in dose of $\sim 10$ cGy and less at the first stage of treatment of oncological diseases (esophageal cancer, breast cancer, carcinoma of lung). At the second stage, irradiation in the therapeutic dose of $1.9 \mathrm{~Gy}$ is administered. In such multistage treatment, the inhibition of cancer growth and tumor's degradation were much more pronounced than in the case when pre-radiation in a low dose had not been performed [2].

Biologically active substances in ultra-low doses (including those used in homeopathy)

Studies and experience have shown that biologically active substances in ultra-low doses increase sensitivity of biological objects to subsequent exposure to intensive physical and chemical factors [3]. This allows one to get a positive therapeutic effect by using lower doses of intensive factors. For example, the complex of such preparations (in homeopathic doses) as hydrastis, chamomilla ipecacuanha, nux vomica, condurango, and carbo vegetabilis, combined with intensive radiotherapy and chemotherapy can heal serious diseases of lymphatic system. Whereas in contrast, the use of only intensive factors in greater doses may result in complete irreversible disability of the whole organism. 


\section{The field-free magnetic vector potential}

This phenomenon can be explained using the following diagram (Figure 1): the beam of quantum entities emitted by a source is split into two beams: $\mathrm{C}_{1}$ and $\mathrm{C}_{2}$. Beam $\mathrm{C}_{1}$ propagates off the solenoid. Beam $\mathrm{C}_{2}$ passes through a magnetized toroidal solenoid. The solenoid is shielded in such a way that outside the substance of the ring, there is no magnetic field; in the place of propagation of beam $\mathrm{C}_{2}$ the field-free magnetic vector potential exists. Both beams of quantum entities $\mathrm{C}_{1}$ and $\mathrm{C}_{2}$ arrive at the entrances of an interferometer. The character of interference rings testifies that the field-free magnetic vector potential affects the wave function phase of quantum entities of beam $\mathrm{C}_{2}$.

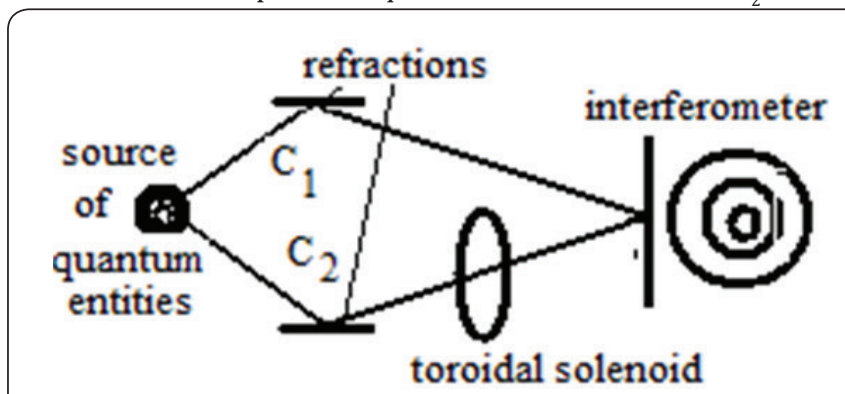

Figure 1 : Schema of the experiment on the effects of field-free magnetic vector potential on quantum entities. $\mathrm{C} 1$ and $\mathrm{C} 2$ are two beams of quantum entities. Beam C2 passes through the shielded magnetized toroidal solenoid. Interference rings are produced by the interferometer.

According to experimental data, field-free magnetic vector potential may influence the properties of biological objects, in particular, those of blood. In the experiments, lymphocytes were recovered from the dark blood of a human using a standard flotation technique based on density gradient. Lymphocyte suspension was exposed at $20-25^{\circ} \mathrm{C}$ to the field-free magnetic vector potential during 60 minutes. After the exposure, the degree of lysis changed versus control by $6 \%$. A change in the blood sedimentation rate (ESR) was observed as well. If the ESR exceeded the standard, it decreased after the exposure; and if it was lower, it became higher after the exposure [4]. That is, in the above experiments the effect of vector potential on blood was "therapeutic".

\section{The cavity structures}

At present there is much evidence of specific influence exerted on biological objects by ambient bodies having a cavity structure (empty bee combs, meshy structures, porous materials, bundles of tubes, pyramids, and many others). For example [5], the healing properties of pyramids are well known. While using nanoparticles in medicine it was observed that AgNP nanoparticles having a triangular form produced a more pronounced toxic effect on Escherichia coli than spherical ones [6].

In Europe, Oskar Korschelt (1853-1940) was likely the first who was granted a patent for the use of specially fabricated cavity structures for medical purposes [7]. In (Figure 2) are shown examples of cavity structures created by Oskar Korschelt. The structure in (Figure 2a) is a tin-plate cylinder with soldered zinc or steel teeth. The structure in (Figure $2 b$ ) is made out of metal wire. The structure in (Figure 2c) is a wooden disk with copper chain coiled on it; it was placed at the ceiling of the room where the healing session took place. The structure in (Figure 2d) was attached to a floor-type setup. By means of these cavity structures Oskar Korschelt treated stomach problems, nerve diseases, insomnia, pains and pneumonia.

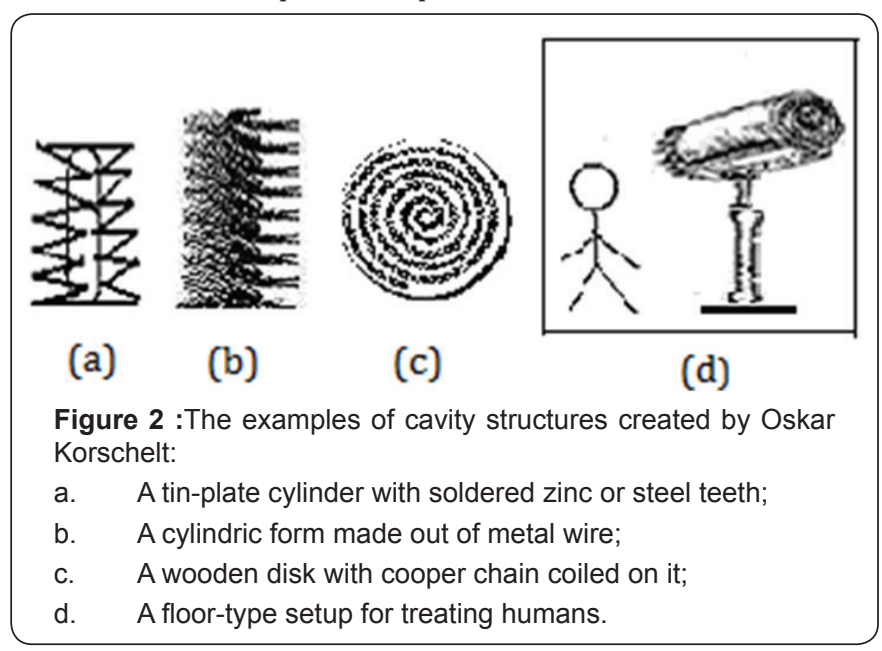

\section{The quantum correlation}

The essence of the phenomenon can be described using the following example. Let two quantum entities (Figure 3) $a$ and $b$ emitted by the same source and having the same wave function at the initial moment of time move in different directions. Entity $a$ is directed, depending on the position (1 or 2) of switch $P$, either towards detector $A_{1}$ or detector $A_{2}$ (these detectors have different characteristics); entity $b$ is directed towards detector $B$. According to postulates of quantum mechanics, the properties of entity $b$ being detected will depend on that which detector detects $a$.

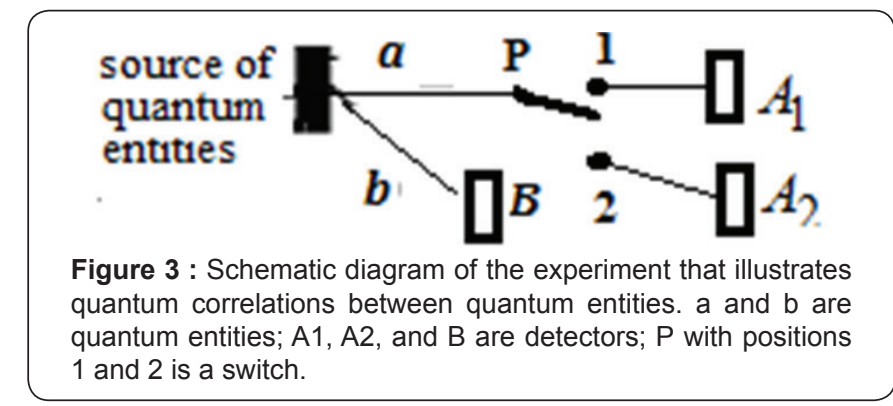

In 1991, Yu. V. Tyagotin carried out an experiment that illustrated quantum correlations between quantum entities of which biological objects consist. The cells grown in the same medium (the cells containing chromosomes of normal splenocyte of mice were used) were divided into two parts. After that one of the parts was placed in noxious conditions. As a result, not only the cells of that part perished, but also perished the cells of other part which were in favorable conditions. [Tyagotin Yu. V. (May 
1993) Communication delivered at the Transregional Scientific Conference "Problems of Biofield" A.S. Popov Scientific and Technical Society, Rostov-Yaroslavsky, Russia]. Thus according to principles of quantum mechanics, it is possible to act upon a diseased organ at a distance. For example, acting upon the blood in a test tube it is possible to act upon the human whose sample of blood was taken.

\section{The physical process underlying the action of ultra- low doses}

The features of the action of low-intensity physical factors (including biologically active substances in ultra-low doses) allows one to make the following conclusions about the physical process underlying such action.

a. The effects of ultra-low doses of biologically active substances and low-intensity radiation on a biological object may be performed by spin supercurrents emerging between virtual particles pairs created in the physical vacuum by quantum entities that constitute, on the one hand, the biological object and, on the other hand, the biologically active substances and radiation [8-9]. The spin supercurrents are similar in their properties to spin supercurrents emerging between spin structures in superfluid ${ }^{3} \mathrm{He}-\mathrm{B}$ [10-11].

b. The physical properties of field-free magnetic vector potential are determined by creation of perturbances by magnetic fields in the physical vacuum (they may be quantum harmonic oscillators possessing zero-point energy). The induction of magnetic field is proportional to the speed of motion of perturbances in the physical vacuum. If this motion is missing but perturbances exist, this means that there is no magnetic field, but magnetic vector potential is present and in this case it is referred to as "field-free magnetic vector potential" [12]. These perturbances may act on spins of virtual particles pairs which are created in the physical vacuum by quantum entities that constitute biological objects.

c. The energetic properties of a cavity structure are determined by spin supercurrents emerging between virtual particles pairs created by quantum entities that constitute the substance of cavity structure. Spin supercurrents may be thought of as "filling" the cavity [13]. The spin supercurrents affect the spins of virtual particles pairs created in the physical vacuum by quantum entities that constitute biological objects.

d. The quantum correlations between quantum entities may be performed by spin supercurrents emerging between virtual particles pairs created by quantum entities [14].

\section{Conclusion}

The decrease of doses of intensive radiotherapy and chemotherapy in treatment of diseases can be done as follows:

i. Before the administering of intensive radiotherapy and chemotherapy it is necessary to perform the irradiation of the diseased organ by low-intensive infra-low-frequency electromagnetic field or/and the treatment by biologically active substances in ultra-low doses.

ii. Affect the diseased organ by the field-free magnetic vector potential.

iii. To follow definite recommendations concerning the forms of the room where the patient resides. The bed of the patient should not be placed in a corner of the room or in the room which is a sort of narrow niche. The ceiling above the bed should not be bent inwards, it is better for it to be dome shaped, as in religious buildings. If the ceiling is flat, a canopy can be arranged over the bed. (It should be noted that the sleeping place of pharaohs and emperors was in the center of room under a canopy.)

iv. It is desirable that preparation of food and care were performed by a patient's healthy relative. Energetic characteristics of quantum entities that constitute the diseased organ of patient may be similar to those of identical organ of a relative. In this case, quantum correlations are possible between the same organs of the diseased person and his/her healthy relative. Thus a correction (healing) of the diseased organ may take place. However, there is a possibility that the patient can "infect" the relative, although the "disease" may be absolutely non-contagious from the standpoint of medical science.

\section{References}

1. Makeev VB, Temuryants NA (1982) Frequency dependence of the biological efficacy of magnetic field in the micropulsation range of geomagnetic field $(0.01-100 \mathrm{~Hz})$. Problemy kosmicheskoi biologii 43: 116-128.

2. Little JB (1990) Low-dose radiation effects: interactions and synergism. Health Phys 59(1): 49-55.

3. Fiedler N, Kipen H (1997) Chemical sensitivity: the scientific literature. Environ Health Perspect 105(2): 409-415.

4. Trukhan EM (2009) Impact of weak electro-magnetic fields on biological activity of water phase. Computer Studies and Simulation 1(1): 101-108.

5. Dimde M (1993) Die Heilkraft der Pyramiden. Die Geheimnisse der AltagyptischenPriester und Heiler. MVG-verlag, a sister company of verlagmoderneindustrie AG \& Co. KG 86895 Landsberg am Lech, Germany.

6. Pal S, Tak YK, Song JM (2007) Does the antibacterial activity of silver nanoparticles depend upon the shape of the nanoparticles? Appl Environ Micro 73(6): 1712-1720.

7. Kaiserliches Patentamt, Patentschrift Nr. 69340, ausgegeben am 22.6.1893 Klasse 30: Gesundheitspflege. Oskar Korschelt in Leipzig. Ein Apparat für therapeutische Zwecke ohne bestimmte oder bewußte Suggestion. Patentiert im Deutschen Reiche vom 14, Juni 1891.

8. Boldyreva LB (2011) An analogy between effects of ultra-low doses of biologically active substances on biological objects and properties of spin supercurrents in superfluid ${ }^{3} \mathrm{He}-\mathrm{B}, \mathrm{Homeopathy} \mathrm{100(3):} \mathrm{187-193.}$ Doi: 10.1016/j.homp.2010.08.007.

9. Boldyreva LB (2013) The Physical Aspect of Action of Biologically Active Substances in Ultra-Low Doses and Low-Intensity Physical 
Factors on Biological Objects: Spin Supercurrents. Altern.Integ Med 2(2): 1000110 (6 pp.)

10. Borovic-Romanov AS, Bunkov YuM, Dmitriev VV, Mukharskii YuM, Sergatskov DA (1989) Investigation of Spin Supercurrents in ${ }^{3} \mathrm{He}-\mathrm{B}$ Phys Rev Lett 62(14): 1631.

11. Fomin IA (1987) Critical superfluid spin current in ${ }^{3} \mathrm{He}-\mathrm{B}$. JETP Lett 45(12): 135-138.

12. Boldyreva LB (2016) The Model of Magnetic Field Based on the
Concepts of Virtual Particles and Quantum Harmonic Oscillators Possessing Zero-Point Energy. International Journal of Physics 4(2): 26-31.

13. Boldyreva LB (2013) The cavity structure effect in medicine: the physical aspect. Forschende Komplementärmedizin /Research in Complementary Medicine 20(5): 322-326.

14. Boldyreva LB (2014) Quantum correlations - Spin supercurrents International Journal of Quantum Information 12(1): 13

This work is licensed under Creative

Commons Attribution 4.0 License

DOI: $10.19080 /$ CTOIJ.2017.05.555671

\section{Your next submission with Juniper Publishers} will reach you the below assets

- Quality Editorial service

- Swift Peer Review

- Reprints availability

- E-prints Service

- Manuscript Podcast for convenient understanding

- Global attainment for your research

- Manuscript accessibility in different formats

( Pdf, E-pub, Full Text, Audio)

- Unceasing customer service

Track the below URL for one-step submission https://juniperpublishers.com/online-submission.php 